\title{
Estimation of Parameters of Johnson's System of Distributions
}

Florence George

Florida International University, fgeorge@fiu.edu

K. M. Ramachandran

University of South Florida, ram@usf.edu

Follow this and additional works at: http://digitalcommons.wayne.edu/jmasm

Part of the Applied Statistics Commons, Social and Behavioral Sciences Commons, and the Statistical Theory Commons

\section{Recommended Citation}

George, Florence and Ramachandran, K. M. (2011) "Estimation of Parameters of Johnson's System of Distributions," Journal of Modern Applied Statistical Methods: Vol. 10 : Iss. 2 , Article 9.

DOI: $10.22237 /$ jmasm/1320120480

Available at: http://digitalcommons.wayne.edu/jmasm/vol10/iss2/9 


\section{Estimation of Parameters of Johnson's System of Distributions}

\author{
Florence George \\ Florida International University \\ Miami, FL
}

\author{
K. M. Ramachandran \\ University of South Florida \\ Tampa, FL
}

Fitting distributions to data has a long history and many different procedures have been advocated. Although models like normal, log-normal and gamma lead to a wide variety of distribution shapes, they do not provide the degree of generality that is frequently desirable (Hahn \& Shapiro, 1967). To formally represent a set of data by an empirical distribution, Johnson (1949) derived a system of curves with the flexibility to cover a wide variety of shapes. Methods available to estimate the parameters of the Johnson distribution are discussed, and a new approach to estimate the four parameters of the Johnson family is proposed. The estimate makes use of both the maximum likelihood procedure and least square theory. The new MLE-Least Square approach is compared with other two commonly used methods. A simulation study shows that the MLE-Least square approach provides better results for $S_{B}, S_{U}$ and $S_{L}$ families.

Key words: Johnson distribution, unbouded, bounded, lognormal, estimation.

Introduction

Any data set with finite moments can be fitted by a member of the Johnson families such as $S_{B}, S_{U}$ or $S_{L}$. The most commonly used methods to estimate the parameters of the Johnson distribution are the percentile approach (Shapiro, 1980) and Quantile method (Wheeler, 1980). A new approach is proposed for the estimation of Johnson parameters and is compard to other methods. For additional reerences, see Drapper (1952), Hill (1976), Hahn and Shapiro (1967), George, et al (2009).

The Johnson Translation System

Given a continuous random variable $X$ whose distribution is unknown and is to be approximated, Johnson proposed three normalizing transformations having the general

Florence George is an Assistant Professor in the Department of of Mathematics and Statistics, Florida International University. Email: fgeorge@fiu.edu. K. M. Ramachandran is a Professor in the Department of Mathematics and Statistics, University of South Florida. Email: ram@usf.edu. form:

$$
Z=\gamma+\delta f\left(\frac{X-\xi}{\lambda}\right)
$$

where $f($.$) denotes the transformation function,$ $Z$ is a standard normal random variable, $\gamma$ and $\delta$ are shape parameters, $\lambda$ is a scale parameter and $\xi$ is a location parameter. Without loss of generality, it is assumed that $\delta>0$ and $\lambda>0$. The first transformation proposed by Johnson defines the lognormal system of distributions denoted by $S_{L}$ :

$$
\begin{aligned}
Z & =\gamma+\delta \ln \left(\frac{X-\xi}{\lambda}\right), X>\xi \\
& =\gamma^{*}+\delta \ln (X-\xi), X>\xi
\end{aligned}
$$

$S_{L}$ curves cover the lognormal family.

The bounded system of distributions $S_{B}$ is defined by

$$
Z=\gamma+\delta \ln \left(\frac{X-\xi}{\xi+\lambda-X}\right), \xi<X<\xi+\lambda
$$


$S_{B}$ curves cover bounded distributions. The distributions can be bounded on the lower end, the upper end or both ends. This family covers Gamma distributions, Beta distributions and many others.

The unbounded system of distributions $S_{U}$ is defined by

$$
\begin{aligned}
Z= & \gamma+\delta \ln \left[\left(\frac{X-\xi}{\lambda}\right)+\left\{\left(\frac{X-\xi}{\lambda}\right)^{2}+1\right\}^{1 / 2}\right], \\
& -\infty<X<\infty \\
= & \gamma+\delta \sinh ^{-1}\left(\frac{X-\xi}{\lambda}\right)
\end{aligned}
$$

The $S_{U}$ curves are unbounded and cover the $t$ and normal distributions, among others. Using the fact that, after the transformation in (2.1), $Z$ follows standard normal distribution, the probability density function (pdf) of each of the family in the Johnson system can be derived. If $X$ follows the Johnson distribution and $Y=\frac{X-\xi}{\lambda}$ then, for $S_{L}$ family, the pdf is

$$
\begin{gathered}
p(y)=\frac{\delta}{\sqrt{2 \pi}} \times \frac{1}{y} \times \exp \left\{-\frac{1}{2}[\gamma+\delta \cdot \ln (y)]^{2}\right\}, \\
\xi<X<+\infty .
\end{gathered}
$$

similarly, for the $S_{B}$ family, the pdf is,

$$
\begin{aligned}
& p(y)= \\
& \frac{\delta}{\sqrt{2 \pi}} \times \frac{1}{[y /(1-y)]} \times \exp \left\{-\frac{1}{2}\left[\gamma+\delta \cdot \ln \left(\frac{y}{1-y}\right)\right]^{2}\right\} \\
& \xi<X<+\xi+\lambda .
\end{aligned}
$$

The pdf for the $S_{U}$ family is

$$
\begin{aligned}
& p(y)= \\
& \frac{\delta}{\sqrt{2 \pi}} \times \frac{1}{\sqrt{y^{2}+1}} \times \exp \left\{-\frac{1}{2}\left[\gamma+\delta \cdot \ln \left(y+\sqrt{y^{2}+1}\right)\right]^{2}\right\}, \\
& -\infty<X<+\infty .
\end{aligned}
$$

In general the pdf of $X$ is given by,

$$
p(x)=\frac{\delta}{\lambda \sqrt{2 \pi}} \times g^{\prime}\left(\frac{x-\xi}{\lambda}\right) \times \exp \left\{-\frac{1}{2}\left[\gamma+\delta \cdot g\left(\frac{x-\xi}{\lambda}\right)\right]^{2}\right\}
$$

for all $x \in H$, where

$$
\begin{aligned}
g^{\prime}(y) & =\frac{1}{y} \text { for the } S_{L} \text { family } \\
& =\frac{1}{[y(1-y)]} \text { for the } S_{B} \text { family } \\
& =\frac{1}{\sqrt{y^{2}+1}} \quad \text { for the } S_{U} \text { family }
\end{aligned}
$$

and

$$
\begin{aligned}
g(y) & =\ln (y) \text { for the } S_{L} \text { family } \\
& =\ln (y /(1-y)) \text { for the } S_{B} \text { family } \\
& =\ln \left[y+\sqrt{y^{2}+1}\right] \text { for the } S_{U} \text { family. }
\end{aligned}
$$

The support $H$ of the distribution is:

$$
\begin{aligned}
H & =[\xi,+\infty) \text { for the } S_{L} \text { family } \\
& =[\xi, \xi+\lambda] \text { for the } S_{B} \text { family } \\
& =(-\infty,+\infty) \text { for the } S_{U} \text { family. }
\end{aligned}
$$

Parameter Estimation of the Johnson System: Percentile Matching

Percentile matching involves estimating $k$ required parameters by matching $k$ selected quantiles of the standard normal distribution with corresponding quantile estimates of the target population. For given percentages $\left\{\alpha_{j}: 1 \leq j \leq k\right\}$, the corresponding quantiles $\left\{z_{\alpha_{j}}\right\}$ and $\left\{x_{\alpha_{j}}\right\}$ are given respectively by 


\section{ESTIMATION OF PARAMETERS OF JOHNSON'S SYSTEM OF DISTRIBUTION}

$$
z_{\alpha_{j}}=\Phi^{-1}\left(\alpha_{j}\right)
$$

and

$$
x_{\alpha_{j}}=F^{-1}\left(\alpha_{j}\right)
$$

where $\Phi($.$) is the standard normal distribution$ function and $F$ is the target distribution function. Once the functional form $f($.$) among$ systems given by equations 2.2-2.4 has been identified, the method of percentile matching attempts to solve the $k$ equations

$$
z_{\alpha_{j}}=\gamma+\delta f\left(\frac{\hat{x}_{\alpha_{j}}-\xi}{\lambda}\right), 1 \leq j \leq k
$$

where $\hat{x}_{\alpha_{j}}$ is an estimator of the quantile $x_{\alpha_{j}}$ based on sample data.

Slifker and Shapiro (1980) introduced a selection rule, which is a function of four percentiles for selecting one of the three families, to give estimates of the Johnson parameters. The fit parameters for the transformation are calculated by solving the transformation equation for the chosen distribution type at the four selected percentiles. Choose any fixed value $z \quad(0<z<1)$ of a standard normal variate; the four points $\pm z$ and $\pm 3 z$ determine three intervals of equal length. Determine the percentile $P_{\zeta}$ corresponding to $\zeta=3 z, z,-z,-3 z$ respectively. For example, if $z=0.5$ then $P_{0.5}=0.6915 * 100=69.15$. Let $x_{3 z}, x_{z}, x_{-z}, x_{-3 z}$ be the percentiles of data values corresponding to the four selected percentiles of the normal distribution. The type of Johnson distribution chosen is based on the value of the discriminant $d$ calculated as follows.

$$
d=\frac{m n}{p^{2}}
$$

where

$$
p=x_{z}-x_{-z}, m=x_{3 z}-x_{z}, n=x_{-z}-x_{-3 z} .
$$

If the calculated discriminant $d$ is greater than 1.001, then an unbounded distribution is chosen; if the value is less than 0.999 , then a bounded distribution is chosen. A discriminant equal to or between the two values results in a lognormal fit. The fit parameters for the transformation are calculated by solving the transformation equation for the chosen distribution type at the four selected percentiles. The parameter estimates for the Johnson $S_{U}$ distribution are:

$$
\hat{\delta}=\frac{2 z}{\cosh ^{-1}\left[\frac{1}{2}\left(\frac{m}{p}+\frac{n}{p}\right)\right]},
$$$$
\hat{\gamma}=\hat{\delta}_{\sinh ^{-1}}\left[\frac{\frac{n}{p}-\frac{m}{p}}{2\left(\frac{m}{p} \frac{n}{p}-1\right)^{1 / 2}}\right],
$$

$$
\hat{\lambda}=\frac{2 p\left(\frac{m}{p} \frac{n}{p}-1\right)^{1 / 2}}{\left(\frac{m}{p}+\frac{n}{p}-2\right)\left(\frac{m}{p}+\frac{n}{p}+2\right)^{1 / 2}},
$$

and

$$
\hat{\xi}=\frac{x_{z}+x_{-z}}{2}+\frac{p\left(\frac{n}{p}-\frac{m}{p}\right)}{2\left(\frac{m}{p}+\frac{n}{p}-2\right)} .
$$

The parameter estimates for the $S_{B}$ distribution are

$$
\hat{\delta}=\frac{z}{\cosh ^{-1}\left(\frac{1}{2}\left[\left(1+\frac{p}{m}\right)\left(1+\frac{p}{n}\right)\right]^{1 / 2}\right)} ;
$$




$$
\begin{gathered}
\hat{\gamma}=\hat{\delta}_{\sinh ^{-1}}\left[\frac{\left.\left(\frac{p}{n}-\frac{p}{m}\right)\left[\left(1+\frac{p}{m}\right)\left(1+\frac{p}{n}\right)-4\right]^{1 / 2}\right]}{2\left(\frac{p}{m} \frac{p}{n}-1\right)}\right], \\
\hat{\lambda}=\frac{p\left[\left\{\left(1+\frac{p}{m}\right)\left(1+\frac{p}{n}\right)-2\right\}^{2}-4\right]^{1 / 2}}{\frac{p}{m} \frac{p}{n}-1},
\end{gathered}
$$

and

$$
\hat{\xi}=\frac{x_{z}+x_{-z}}{2}-\frac{\lambda}{2}+\frac{p\left(\frac{p}{n}-\frac{p}{m}\right)}{2\left(\frac{p}{m} \frac{p}{n}-1\right)}
$$

The parameter estimates for the Johnson $S_{L}$ distribution are:

$$
\begin{gathered}
\hat{\delta}=\frac{2 z}{\ln \left(\frac{m}{p}\right),} \\
\hat{\gamma}^{*}=\hat{\delta} \ln \left[\frac{\frac{m}{p}-1}{p\left(\frac{m}{p}\right)^{1 / 2}}\right],
\end{gathered}
$$

and

$$
\hat{\xi}=\frac{x_{z}+x_{-z}}{2}-\frac{p}{2} \frac{\frac{m}{p}+1}{\frac{m}{p}-1} .
$$

Parameter Estimation of the Johnson System: Quantile Estimators

Wheeler (1980) proposed a method to estimate the parameters $\gamma$ and $\delta$ in the Johnson family using five quantiles. Let $p_{n}=\left(n-\frac{1}{2}\right) / n$, where $n$ is the sample size. Denote the quantile of the standard normal distribution corresponding to the cumulative probability $p_{n}$ by $z_{n}$. For example, if $n=100$, then $p_{n}=0.995$, so that $z_{n}=2.5758$. Choose five quantiles $x_{p}, x_{k}, x_{0}, x_{m}, x_{n}$ from data corresponding to standard normal quantiles $z=-z_{n},-\frac{1}{2} z_{n}, 0, \frac{1}{2} z_{n}, z_{n}$. The general form of the Johnson system can be written as

$$
z=\gamma+\delta \ln f(y)
$$

$$
\begin{array}{llll}
\text { where } \quad f(y)=y & \text { for } & S_{L}, \\
f(y)=y+\left(1+y^{2}\right)^{1 / 2} ; & \text { for } & S_{U}, \\
f(y)=y /(1-y) ; & \text { and for } & S_{B} & y=(x-\xi) / \lambda .
\end{array}
$$
Wheeler uses the fact that any quantity of the form

$$
\frac{x_{i}-x_{j}}{x_{r}-x_{s}}=\frac{f^{-1}\left(\omega_{i}\right)-f^{-1}\left(\omega_{j}\right)}{f^{-1}\left(\omega_{r}\right)-f^{-1}\left(\omega_{s}\right)}
$$

where $\omega=e^{(z-\gamma) / \delta}$, does not depend on $\xi$ or $\lambda$. The parameter estimates for the $S_{U}$ curves are:

$$
\hat{\delta}=\frac{1}{2} z_{n} / \ln b
$$

where

$$
b=\frac{1}{2} t_{u}+\left[\left(\frac{1}{2} t_{u}\right)^{2}-1\right]^{1 / 2},
$$

and

$$
t_{u}=\frac{x_{n}-x_{p}}{x_{m}-x_{k}}
$$

and

$$
\hat{\gamma}=-\delta \ln (a)
$$

where

$$
a^{2}=\frac{1-t b^{2}}{t-b^{2}} \text { and } t=\frac{x_{n}-x_{0}}{x_{0}-x_{p}} .
$$

For $S_{B}$ curves the parameter estimates are: 


$$
\hat{\delta}=\frac{1}{2} z_{n} / \ln b
$$

where

$$
b=\frac{1}{2} t_{b}+\left[\left(\frac{1}{2} t_{b}\right)^{2}-1\right]^{1 / 2}
$$

and

$$
\begin{gathered}
t_{b}=\frac{\left(x_{m}-x_{0}\right)\left(x_{n}-x_{p}\right)}{\left(x_{n}-x_{m}\right)\left(x_{0}-x_{p}\right)}, \\
\hat{\gamma}=-\delta \ln (a),
\end{gathered}
$$

where

$$
a=\frac{t-b^{2}}{1-t b^{2}} \text { and } t=\frac{x_{n}-x_{0}}{x_{0}-x_{p}} .
$$

For $S_{L}$ curves,

$$
\hat{\delta}=\frac{z_{n}}{\ln t}
$$

where

$$
t=\frac{x_{n}-x_{0}}{x_{0}-x_{p}}
$$

To differentiate the three types of Johnson curves, the ratio

$$
\frac{t_{b}}{t_{u}}=\frac{\left(x_{m}-x_{0}\right)\left(x_{m}-x_{k}\right)}{\left(x_{n}-x_{m}\right)\left(x_{0}-x_{p}\right)}
$$

is used. It is less than 1 for $s_{U}$, equal to 1 for $S_{L}$ and greater than 1 for $S_{B}$.

Parameter Estimation of the Johnson System: Proposed MLE-Least Square Approach

A new algorithm to estimate parameters of Johnson's distribution is now proposed; this algorithm is named the MLE-Least Square Approach, because both maximum likelihood and least square approaches were employed to estimate the four parameters. Although the maximum likelihood equations for $\gamma$ and $\delta$ were derived by Storer (1987), there are no closed form solutions for $\xi$ and $\lambda$. The idea of combining both a maximum likelihood approach and least square theory makes the derivation of all four parameters more tractable analytically.

The probability density functions of the members of the Johnson family are known. First consider the $S_{U}$ and $S_{B}$ family of the Johnson system. Using the general form of Johnson densities (see equation 2.5), the likelihood function is:

$$
L(x)=\frac{\delta^{n}}{\lambda^{n}(2 \pi)^{n / 2}} \prod_{i=1}^{n} g^{\prime}\left(\frac{x-\xi}{\lambda}\right) e^{-\frac{1}{2} \sum_{i=1}^{n}\left(\gamma+\delta g\left(\frac{x-\xi}{\lambda}\right)\right)^{2}},
$$

and the log-likelihood is,

$$
\begin{aligned}
\log L= & n \log \delta-n \log \lambda-n / 2 \log (2 \pi) \\
& +\sum_{i=1}^{n} g^{\prime}\left(\frac{x-\xi}{\lambda}\right)-\frac{1}{2} \sum_{i=1}^{n}\left(\gamma+\delta g\left(\frac{x-\xi}{\lambda}\right)\right)^{2}
\end{aligned}
$$

Setting the partial derivatives with respect to $\delta$ to zero,

$$
\frac{n}{\delta}-\delta \sum\left[g\left(\frac{x-\xi}{\lambda}\right)\right]^{2}-\gamma \sum g\left(\frac{x-\xi}{\lambda}\right)=0
$$

which can be written as,

$$
\delta^{2} \sum\left[g\left(\frac{x-\xi}{\lambda}\right)\right]^{2}+\gamma \delta \sum g\left(\frac{x-\xi}{\lambda}\right)-n=0
$$

Setting the partial derivatives with respect to $\gamma$ to zero,

$$
n \gamma+\delta \sum g\left(\frac{x-\xi}{\lambda}\right)=0
$$

which yields,

$$
\begin{aligned}
\hat{\gamma} & =\frac{-\delta \sum g\left(\frac{x-\xi}{\lambda}\right)}{n} . \\
& =-\delta \bar{g}
\end{aligned}
$$

Using (3.3) in (3.2): 


$$
\begin{aligned}
\hat{\delta}^{2} & =\frac{n}{\sum\left[g\left(\frac{x-\xi}{\lambda}\right)\right]^{2}-\frac{1}{n}\left[\sum g\left(\frac{x-\xi}{\lambda}\right)\right]^{2}} \\
& =\frac{1}{\operatorname{var}(g)}
\end{aligned}
$$

where $\bar{g}$ is the mean and $\operatorname{var}(g)$ is the variance of the values of $g$ defined in (2.6).

The partial derivatives of the loglikelihood with respect to $\xi$ and $\lambda$ are not simple. Storer (1987) presents a lengthy strategy for obtaining the solutions of these parameters. In the maximum likelihood estimation method, Kamziah, et al. (1999) applied the NewtonRaphson iteration to maximize the log likelihood of the Johnson distribution. They observed that, for some samples, the log likelihood function does not have a local maximum with respect to parameters $\xi$ and $\lambda$. This non-regularity of the likelihood function caused occasional nonconvergence of the Newton-Raphson iteration that was used to maximize the log-likelihood (Hosking, 1985)

The least squares method is applied herein to estimate parameters $\xi$ and $\lambda$. From (2.1), $x=\xi+\lambda f^{-1}\left(\frac{z-\gamma}{\delta}\right)$ is obtained. For

fixed values of $\gamma$ and $\delta$, this equation may be considered as a linear equation with parameters $\xi$ and $\lambda$.

The sum of squares of errors is,

$$
S(\xi, \lambda)=\sum\left[x-\xi+\lambda f^{-1}\left(\frac{z-\gamma}{\delta}\right)\right]^{2} .
$$

To determine the value of $\xi$ and $\lambda$ that minimizes $S(\xi, \lambda)$, the partial derivatives of $S(\xi, \lambda)$ with respect to $\xi$ and $\lambda$ are calculated and these partial derivatives are equated to zero. The following two equations, called normal equations, are then obtained:

$$
\sum x=n \xi+\lambda \sum f^{-1}\left(\frac{z-\gamma}{\delta}\right)
$$

$$
\sum x f^{-1}\left(\frac{z-\gamma}{\delta}\right)=\xi \sum f^{-1}\left(\frac{z-\gamma}{\delta}\right)+\lambda \sum\left[f^{-1}\left(\frac{z-\gamma}{\delta}\right)\right]^{2}
$$

Note that $z$ is a standard normal variate. The quantiles of $x$ and the corresponding quantiles of $z$ can be considered paired observations. If there are 100 or more $x$ values, the percentiles 1 through 99 would be considered. If the number of data points of $x$ is $k$ where $k$ is less than $100, k-1$ quantiles of $x$ and the corresponding $k-1$ quantiles of $z$ would be considered as paired observations.

Solving the normal equations results in

$$
\hat{\lambda}=\frac{n \sum x f^{-1}\left(\frac{z-\gamma}{\delta}\right)-\sum f^{-1}\left(\frac{z-\gamma}{\delta}\right) \sum x}{n \sum\left[f^{-1}\left(\frac{z-\gamma}{\delta}\right)\right]^{2}-\left[\sum f^{-1} \frac{z-\gamma}{\delta}\right]^{2}}
$$

and

$$
\hat{\xi}=\bar{x}-\lambda * \operatorname{mean}\left[f^{-1}\left(\frac{z-\gamma}{\delta}\right)\right]
$$

where $\bar{x}$ is the mean of $x$-quantiles and $\bar{z}$ is the mean of $z$-quantiles used in the above equations. Starting with some initial values of $\xi$ and $\lambda$, these initial values may be taken as the estimates obtained by any one of the previous methods. The estimates of $\gamma$ and $\delta$ are then calculated using equations (3.2) and (3.3). After the estimates of $\gamma$ and $\delta$ are obtained, equations (3.5) and (3.6) can be used to revise the $\xi$ and $\lambda$ estimates. Now these steps may be repeated, each time using the most recent estimates; the Residual Sum of Squares(RSS) can be tracked and, after a few steps, the estimate with minimum RSS value selected.

For the $S_{L}$ family, consider the transformation in equation (2.2), so that there are only 3 parameters included. The probability density function can be given by,

$$
p(x)=\frac{\delta}{\sqrt{2 \pi}} \frac{1}{(x-\xi)} e^{-\frac{1}{2}\left[\gamma^{*}+\delta \ln (x-\xi)\right]^{2}}
$$

The likelihood function is, 


$$
L(x)=\frac{\delta^{n}}{(2 \pi)^{n / 2}} \frac{1}{\prod(x-\xi)} e^{-\frac{1}{2} \sum\left[\gamma^{*}+\delta \ln (x-\xi)\right]^{2}}
$$

Setting the partial derivative of log-likelihood with respect to $\delta$ to zero we get,

$$
\frac{n}{\delta}-\delta \sum[\ln (x-\xi)]^{2}-\gamma^{*} \sum[\ln (x-\xi)]=0
$$

which can be written as,

$$
\delta^{2} \sum[\ln (x-\xi)]^{2}+\gamma^{*} \delta \sum[\ln (x-\xi)]-n=0
$$

Setting the partial derivative of log-likelihood with respect to $\gamma^{*}$ to zero,

$$
n \gamma^{*}+\delta \sum[\ln (x-\xi)]=0
$$

which gives,

$$
\begin{aligned}
\hat{\gamma}^{*} & =-\frac{1}{n} \delta \sum[\ln (x-\xi)] \\
& =-\delta \bar{g}^{*} .
\end{aligned}
$$

Using (3.9) in (3.8) and solving for $\delta$, results in

$$
\begin{aligned}
\hat{\delta}^{2} & =\frac{n}{\sum[\ln (x-\xi)]^{2}-\frac{\left[\sum \ln (x-\xi)\right]^{2}}{n}} \\
& =\frac{1}{\operatorname{var}\left(g^{*}\right)}
\end{aligned}
$$

where $g^{*}=\ln (x-\xi)$. To estimate $\xi$, as before, use the method of least squares in the equation

$$
x=\xi+f^{-1}\left(\frac{z-\gamma^{*}}{\delta}\right) .
$$

The sum of squares of errors is,

$$
S(\xi)=\sum\left(x-\xi+f^{-1}\left(\frac{z-\gamma^{*}}{\delta}\right)\right)^{2}
$$

To find the value of $\xi$ that minimizes $S(\xi)$, obtain

$$
\frac{d S}{d \xi}=-2 \sum\left(x-\xi-f^{-1}\left(\frac{z-\gamma^{*}}{\delta}\right)\right)
$$

Setting this derivative equal to zero, results in:

$$
\hat{\xi}=\bar{x}-\operatorname{mean}\left[f^{-1}\left(\frac{z-\gamma^{*}}{\delta}\right)\right]
$$

Here the same situation arises, the estimate of $\xi$ depends on $\gamma^{*}$ and $\delta$ and vice versa; as in the case of the $S_{U}$ and $S_{B}$ distributions. Thus, start with some initial value of $\xi$ to estimate $\gamma^{*}$ and $\delta$, then use these estimated values to estimate $\xi$. Repeat this procedure, keeping track of RSS, and choose the one with least RSS.

\section{Results}

Data of size 2,000 were simulated from the $S_{U}$, $S_{B}$ and $S_{L}$ distributions to compare different methods of estimation. Twenty samples of size 2,000 were generated from each of the three specified models. The mean and the Mean Square Error (MSE) of the estimated values of the $S_{B}, S_{U}$, and $S_{L}$ families are shown in Tables 1, 2 and 3. It can be observed that the average of the estimates are close to the true values of the parameters and, in general, the MSE of the estimates are smaller in the proposed method than the other methods.

\section{Conclusion}

A new approach that makes use of both the maximum likelihood procedure and least square theory was proposed to estimate the four parameters of the Johnson family of distributions. The new MLE-Least Square approach is compared with two other commonly used methods. The simulation study shows that the MLE-Least square approach gives better results for the $S_{B}, S_{U}$ and $S_{L}$ families. The findings of this study should be useful for applied practitioners. 
GEORGE \& RAMACHANDRAN

Table 1: Mean and (Mean Square Error-MSE) of Parameter Estimates for the Johnson $S_{B}$ Family

\begin{tabular}{|c|c|c|c|c|c|}
\hline S1. No. & Parameter & $\begin{array}{l}\text { True } \\
\text { Value }\end{array}$ & $\begin{array}{l}\text { Percentile } \\
\text { Method }\end{array}$ & $\begin{array}{l}\text { Quantile } \\
\text { Method }\end{array}$ & $\begin{array}{c}\text { MLE-Least } \\
\text { Square Approach }\end{array}$ \\
\hline \multirow{4}{*}{1} & $\gamma$ & 1 & $0.998(0.167)$ & $1.063(0.409)$ & $0.997(0.026)$ \\
\hline & $\delta$ & 1 & $1.001(0.059)$ & $1.024(0.083)$ & $0.997(0.026)$ \\
\hline & $\xi$ & 10 & $10.047(0.085)$ & $9.982(0.131)$ & $9.93(0.08)$ \\
\hline & $\lambda$ & 10 & $10.049(5.92)$ & $10.402(14.37)$ & $10.57(4.99)$ \\
\hline \multirow{4}{*}{2} & $\gamma$ & 0.5 & $0.503(0.009)$ & $0.503(0.0493)$ & $0.494(0.007)$ \\
\hline & $\delta$ & 0.5 & $0.505(0.003)$ & $0.519(0.023)$ & $0.507(0.001)$ \\
\hline & $\xi$ & 10 & $9.11(4.038)$ & $9.97(0.077)$ & $10.004(0.004)$ \\
\hline & $\lambda$ & 10 & $10.005(0.285)$ & $10.094(1.614)$ & $9.868(2.056)$ \\
\hline \multirow{4}{*}{3} & $\gamma$ & 1 & $1.032(0.065)$ & $1.01(0.015)$ & $1.016(0.017)$ \\
\hline & $\delta$ & 0.5 & $0.507(0.0039)$ & $0.5006(0.0013)$ & $0.509(0.002)$ \\
\hline & $\xi$ & 10 & 9.698(.488) & $10.001(0.001)$ & $10.001(0.001)$ \\
\hline & $\lambda$ & 10 & $10.355(4.63)$ & $10.085(0.69)$ & $9.86(0.70)$ \\
\hline \multirow{4}{*}{4} & $\gamma$ & 0.5 & $0.558(0.287)$ & $0.539(0.136)$ & $0.561(0.165)$ \\
\hline & $\delta$ & 1 & $1.013(0.191)$ & $1.024(0.108)$ & $1.055(0.115)$ \\
\hline & $\xi$ & 10 & $9.82(1.097)$ & $9.94(0.55)$ & $9.91(0.52)$ \\
\hline & $\lambda$ & 10 & $10.31(15.4)$ & $10.30(8.2)$ & $9.83(0.50)$ \\
\hline
\end{tabular}


ESTIMATION OF PARAMETERS OF JOHNSON'S SYSTEM OF DISTRIBUTION

Table 2: Mean and (Mean Square Error-MSE) of Parameter Estimates for the Johnson $S_{U}$ Family

\begin{tabular}{|c|c|c|c|c|c|}
\hline Sl. No. & Parameter & $\begin{array}{l}\text { True } \\
\text { Value }\end{array}$ & $\begin{array}{l}\text { Percentile } \\
\text { Method }\end{array}$ & $\begin{array}{l}\text { Quantile } \\
\text { Method }\end{array}$ & $\begin{array}{c}\text { MLE-Least } \\
\text { Square Approach }\end{array}$ \\
\hline \multirow{4}{*}{1} & $\gamma$ & 0 & $0.04(0.32)$ & $0.015(0.05)$ & $0.015(0.05)$ \\
\hline & $\delta$ & 2 & $1.41(3.3)$ & $2.08(0.34)$ & $2.05(0.29)$ \\
\hline & $\xi$ & 10 & $10.24(8.9)$ & $10.1(1.5)$ & $10.1(1.4)$ \\
\hline & $\lambda$ & 10 & $12.3(99.9)$ & $10.5(12.6)$ & $10.3(10.1)$ \\
\hline \multirow{4}{*}{2} & $\gamma$ & 0.5 & $0.82(2.9)$ & $0.52(0.11)$ & $0.51(0.09)$ \\
\hline & $\delta$ & 2 & $2.47(3.23)$ & $2.08(0.45)$ & $2.06(0.37)$ \\
\hline & $\xi$ & 10 & $11.51(64.6)$ & $10.06(2.79)$ & $10.04(2.59)$ \\
\hline & $\lambda$ & 10 & $12.07(56.5)$ & $10.35(12.6)$ & $10.25(11.22)$ \\
\hline \multirow{4}{*}{3} & $\gamma$ & 0 & $-0.003(0.003)$ & $0.005(0.002)$ & $0.003(0.002)$ \\
\hline & $\delta$ & 1 & $1.033(0.006)$ & $0.99(0.003)$ & $0.99(0.002)$ \\
\hline & $\xi$ & 10 & $10.03(.43)$ & $10.05(0.25)$ & $10.06(0.25)$ \\
\hline & $\lambda$ & 10 & $10.45(1.43)$ & $9.82(0.7)$ & $9.75(0.73)$ \\
\hline \multirow{4}{*}{4} & $\gamma$ & 0.5 & $0.514(0.009)$ & $0.488(0.006)$ & $0.487(0.007)$ \\
\hline & $\delta$ & 1 & $1.008(0.006)$ & $0.999(0.006)$ & $0.996(0.006)$ \\
\hline & $\xi$ & 10 & $10.243(1.203)$ & $9.95(0.9)$ & $9.94(1.05)$ \\
\hline & $\lambda$ & 10 & $10.06(0.96)$ & $10.06(1.13)$ & $10.02(1.43)$ \\
\hline
\end{tabular}




\section{GEORGE \& RAMACHANDRAN}

Table 3: Mean and (Mean Square Error-MSE) of Parameter Estimates for the Johnson $S_{L}$ Family

\begin{tabular}{|c|c|c|c|c|c|}
\hline S1. No. & Parameter & $\begin{array}{l}\text { True } \\
\text { Value }\end{array}$ & $\begin{array}{l}\text { Percentile } \\
\text { Method }\end{array}$ & $\begin{array}{l}\text { Quantile } \\
\text { Method }\end{array}$ & $\begin{array}{c}\text { MLE-Least } \\
\text { Square Approach }\end{array}$ \\
\hline \multirow{3}{*}{1} & $\begin{array}{c}\gamma^{*} \\
(\gamma, \lambda)\end{array}$ & $\begin{array}{l}1.303 \\
(1,10)\end{array}$ & $-1.353(0.051)$ & $-1.29(0.027)$ & $1.303(0.04)$ \\
\hline & $\delta$ & 1 & $1.012(0.006)$ & $0.97(0.008)$ & $1.012(0.008)$ \\
\hline & $\xi$ & 0 & $-0.98(0.14)$ & $0.53(0.057)$ & $0.53(0.057)$ \\
\hline \multirow{3}{*}{2} & $\begin{array}{c}\gamma^{*} \\
(\gamma, \lambda)\end{array}$ & $\begin{array}{c}-2.3 \\
(0,10)\end{array}$ & $-2.24(0.04)$ & $-2.26(0.01)$ & $-2.21(0.07)$ \\
\hline & $\delta$ & 1 & $0.98(0.003)$ & $0.98(0.002)$ & $0.98(0.007)$ \\
\hline & $\xi$ & 0 & $0.18(0.41)$ & $0.22(0.36)$ & $0.33(0.28)$ \\
\hline \multirow{3}{*}{3} & $\begin{array}{c}\gamma^{*} \\
(\gamma, \lambda)\end{array}$ & $\begin{array}{l}-5.91 \\
(1,10)\end{array}$ & $-6.53(22.9)$ & $-5.26(18.13)$ & $-5.47(12.36)$ \\
\hline & $\delta$ & 3 & $3.18(2.28)$ & $2.66(3.66)$ & $2.87(1.42)$ \\
\hline & $\xi$ & 0 & $-0.503(15.28)$ & $0.72(18.3)$ & $0.504(7.17)$ \\
\hline \multirow{3}{*}{4} & $\begin{array}{c}\gamma^{*} \\
(\gamma, \lambda)\end{array}$ & $\begin{array}{l}-3.45 \\
(1,10) \\
\end{array}$ & $-3.78(3.26)$ & $-3.45(0.99)$ & $-3.45(1.63)$ \\
\hline & $\delta$ & 2 & $2.06(0.35)$ & $1.88(0.35)$ & $1.97(0.21)$ \\
\hline & $\xi$ & 0 & $-0.13(4.12)$ & $0.43(4.41)$ & $0.29(1.67)$ \\
\hline
\end{tabular}

Acknowledgment

The authors are grateful to Dr. B. M. Golam Kibria for his valuable and constructive comments which improved the presetantion of the study.
References

Draper, J. (1952). Properties of distributions resulting from certain simple transformations of the normal distribution. Biometrika, 39, 290-301. 


\section{ESTIMATION OF PARAMETERS OF JOHNSON'S SYSTEM OF DISTRIBUTION}

George, F., Ramachandran, K. M., \& Lihua, L. (2009). Gene selection with Johnson distribution. Journal of Statistical Research, 43, 117-125.

Hahn, J. G., \& Shapiro S. S. (1967). Statistical models in engineering. John Wiley \& Sons, New York.

Hill, I. D., Hill, R., \& Holder, R. L. (1976). Fitting Johnson curvesby moments. Applied Statistics, 25 180-189.

Hosking, J. R. M., Wallis J. R., \& Wood E. F. (1985). Estimation of the generalized extreme-value distribution by the method of probability-weighted moments. Technometrics, 27 251-261.

Johnson, N. L. (1949). Systems of frequency curves generated by methods of translation. Biometrika, 36, 149-176.
Kamziah, A. K., Ahmad, M. I, \& Jaffirin, L. (1999). Nonlinear regresion approach to estimating Johnson SB parameters for diameter data. Canadian Journal of Forestry Resources, 29, 310-314.

Slifker, J., \& Shapiro, S. (1980). The Johnson system: Selection and parameter estimation. Technometrics, 22, 239-247.

Storer, R. H. (1987). Adaptive estimation by maximum likelihood: Fitting of Johnson distributions. Unpublished Ph.D. thesis, School of Industrial and Systems Engineering, Georgia Institue of Technology.

Wheeler, R. (1980). Quantile Estimators of Johnson curve Parameters. Biometrika, 67, 725-728. 